University of Nebraska - Lincoln

DigitalCommons@University of Nebraska - Lincoln

Robert Katz Publications

Research Papers in Physics and Astronomy

January 1991

Radial Distribution of Dose

Robert Katz

University of Nebraska-Lincoln, rkatz2@unl.edu

Matesh N. Varma

U.S. Department of Energy, Washington, D.C.

Follow this and additional works at: https://digitalcommons.unl.edu/physicskatz

Part of the Physics Commons

Katz, Robert and Varma, Matesh N., "Radial Distribution of Dose" (1991). Robert Katz Publications. 115. https://digitalcommons.unl.edu/physicskatz/115

This Article is brought to you for free and open access by the Research Papers in Physics and Astronomy at DigitalCommons@University of Nebraska - Lincoln. It has been accepted for inclusion in Robert Katz Publications by an authorized administrator of DigitalCommons@University of Nebraska - Lincoln. 


\title{
Radial Distribution of Dose
}

\author{
Robert Katz ${ }^{(a)}$ and Matesh N. Varma ${ }^{(b)}$
}

\begin{abstract}
The radial distribution of dose about the path of a heavy ion, principally from delta rays, is one of the central contributions of atomic physics to the systematization of high LET radiation effects in condensed matter, whether the detection arises in chemical, physical, or biological systems. In addition to the radial distribution of dose, we require knowledge of the response of the system to X-rays or gamma-rays or to beams of energetic electrons such that the electron slowing-down spectra from these radiations can approximate the slowing-down spectra from delta rays even at different radial distances from the ion's path. A combination of these data enables us to calculate the action cross sections for heavy ion bombardments in all detectors for which this information is available. These cross sections are indispensable for the evaluation of effects caused by high LET radiations. In this paper we focus attention principally on the calculation and measurement of the radial distribution of dose and on their limitations.
\end{abstract}

\section{Introduction}

The first application of the radial dose distribution in any detector was made by Katz and Butts $(1,2)$ in the study of the width of heavy ion tracks in electron-sensitive nuclear emulsions.

Earlier work had called attention to the significance of delta rays in track production but had assigned responsibility either to delta-ray flux ${ }^{(3)}$ or to energy flux, ${ }^{(4)}$ such that the track width was determined either by an appropriate flux of delta rays or of energy through the region bounding the "track width." While these criteria approximated the width of the tracks of heavy ions in the "thin-down" region in the stopping end of a particle track, neither criterion yielded a correct value of the track width over the entire range of a heavy ion. We calculated the radial distribution of dose, based on the initial energy flux calculations of Bizetti and Della Corte, ${ }^{(4)}$ and revised the track-width criterion to one of a critical radial dose, which would define the "width" of the heavy ion track. This criterion was much more consistent with our trackwidth measurements over the entire range of heavy particle tracks than either of the two earlier criteria. We had identified the radial dose as a quantity upon which to base models of track effects.

\section{Dry Enzymes and Viruses}

In 1967, our calculations of the radial dose distribution were simplified. They were then based on the Rutherford equation for delta-ray production by heavy ions from a sea of free electrons. ${ }^{(5)}$ Additionally, assumptions were made that delta rays were

(a) University of Nebraska, Lincoln, Nebraska

(b) U.S. Department of Energy, Washington, D.C. 
normally ejected, and that the range energy relation for electrons was linear, based on experimental data in aluminum for electrons of energies in the range of delta-ray energies from ions of energy less than about $8 \mathrm{MeV} / \mathrm{amu}$. For heavy ions, we made use of a formula for effective charge as a function of ion velocity from Barkas, ${ }^{(6)}$, which came from measurements of the range of ions from the HILAC accelerator in nuclear emulsion. These results formed the basis of the calculation of the inactivation cross section for dry enzymes and viruses, taken to be 1-hit detectors, and provided the basic structure that underlay many further developments in track theory. ${ }^{(7)}$ Although at that time we had no direct experimental verification of our calculations of radial dose, we considered our calculation of the response of dry enzymes and viruses to energetic heavy ions to be an experimental confirmation of the overall procedure.

Here, we assumed that the average radial dose distribution dominated the calculation of action cross section with no need for detailed knowledge of the fluctuation in energy deposition which later became the theme of the theory of Dual Radiation Action. $(8,9)$ We found that the significance of target size varied. With fast protons and insensitive detectors, size might be significant. There, we may be dealing with thin tracks and relatively large targets. With high LET radiations, we were dealing with thick tracks and small targets rather than the converse, so target size was of negligible importance. We found that earlier preoccupation with LET as a reference variable had been excessive, and that a much better variable was $z^{* 2} / \beta^{2}$. And we found that there must be "hooks" in double-log plots of $\sigma$ versus LET, corresponding to "thindown" of the tracks of heavy ions in nuclear emulsions as the ions approached the end of their range. Many of these observations have since been shown to apply to other physical detectors and to radiobiology. They were signaled by our innovative use of the average radial distribution of dose from delta rays as the dominating theme in a track structure model, ${ }^{(7)}$ and by our use of the detector's response to gamma rays as the means of calibrating the response to heavy ions. To experimenters it said that high LET response should be measured with track-segment irradiation with heavy ions (not neutrons) and that if any model were to emerge from such measurements, they should be accompanied by measurements with gamma rays.

In later work, we have repeatedly used this sort of test of our dose calculations. Our test data have been the measured responses of detectors whose sensitive elements are of different sizes and sensitivities, and with bombardments of a range of ion charges and speeds to check on dose calculations in different regions of radial distance.

\section{Particle Tracks in Emulsions}

In an attempt to provide a firmer basis for the calculation of the radial distribution of dose, data for the range and energy dissipation of beams from electrons normally incident on different materials were systematized analytically. ${ }^{(10,11)}$ That information was then applied to data on the blackness of emulsion exposed to beams of electrons $^{(12)}$ as a test of both the energy dissipation formulas and of the 1-hit model of detector response. It was applied to the calculation of the radial distribution of dose $\mathrm{e}^{(10)}$ in different materials. Of primary importance was the application to a new model of particle tracks in emulsion, where the calculations ${ }^{(13)}$ could be compared with measurements of the grain-count regime. ${ }^{(6,14)}$ The calculations were also compared with microdensitometric measurements of the blackness of emulsion as a function of distance from the ion's path in the track-width regime of very heavy cosmic rays, from iron to uranium. ${ }^{(15)}$ The agreement of our calculations with measurement supported the validity of our calculations of the radial dose distribution, as well as our 
assumption that electron-sensitive emulsions were one-hit detectors. Simulations of particle tracks in nuclear emulsions making use of track theory are compared to photographs of particle tracks to good effect. $(16,17)$

At this time, thus, we had made analytical estimates of the radial distribution of dose in condensed matter ${ }^{(18)}$ based on various approximations to the electron-energy dissipation in condensed matter and on an extension of the Rutherford formula for delta-ray production. We had tested these in application to the response of condensed-phase detectors to energetic ions. We continue to search for improved analytical expressions for the radial dose distribution in gases and in condensed matter, and to test these distributions with calculations of detector response.

Except for microdensitometric measurements of the blackness of nuclear emulsions as a function of transverse distance from the ion's path, ${ }^{(15,19-22)}$ there is no possibility for direct measurement of the radial-dose distribution in solids or liquids.

\section{Experiment}

Initially stimulated by the work of Butts and Katz, a program of measurement of the radial dose distribution in gases was initiated by John Baum and co-workers at the Brookhaven National Laboratory in 1967. In the period from 1967 to 1980, a number of papers were published by this group, initially under Baum's leadership and subsequently under the leadership of Matesh Varma, with participation from S. L. Stone, A. V. Kuehner, C. L. Wingate, and J. T. Lyman. (23-31) Measurements were made of the radial dose distributions in hydrogen, nitrogen, and in tissueequivalent gas, using the following projectiles: protons at 1 and $3 \mathrm{MeV} / \mathrm{amu}$, helium at $0.75,18.3$, and $230 \mathrm{MeV} / \mathrm{amu}$, oxygen at $2.4 \mathrm{MeV} / \mathrm{amu}$, neon at $377 \mathrm{MeV} / \mathrm{amu}$, bromine at $0.53 \mathrm{MeV} / \mathrm{amu}$, and iodine at 0.26 and $0.49 \mathrm{MeV} / \mathrm{amu}$. The slower projectiles were obtained at the Brookhaven National Laboratory using the Tandem Van de Graaf accelerator, while the more energetic projectiles were obtained at the Lawrence Radiation Laboratory using the 184-in. cyclotron and the BEVALAC accelerators. Some accompanying Monte Carlo calculations were made by H. G. Paretzke.

This group used essentially the same equipment for all its measurements. A collimated beam of ions was passed through a gas-filled tank. The resulting ionization was measured in a cylindrical ion chamber whose axis was parallel to, and displaced from, the beam. Together with the effect of varied pressure in the tank, one could simulate the effect of radial distance variation in a medium of constant density. A "W" value (energy per ion pair), based on ion chamber measurements with gamma rays and assumed to be constant over all radial distances, was used to convert ionization density to energy density or dose. Over a substantial range of radial distances, ions, and ion speeds, it was found that the dose varied with the square of the effective charge and inversely with the square of the product of radial distance and ion speed. These pioneering measurements provided significant support to track-structure theory. The measurements were in agreement with our calculations (see Fig. 3) and provided an important test of analytic formulations.

There are, however, some inherent difficulties with these (and other) experiments. It is impossible to approach arbitrarily close to an ion's path. It is virtually impossible to measure the dose out to the radial limit in energy deposition. Since the distribution in electron energies must be expected to vary as a function of radial distance, the W value used to convert ionization density to energy deposition should vary correspondingly. But experimentally, we do not know the electron-energy spectrum as a function of radial distance. These limitations in our knowledge at the smallest and greatest radial distances are not readily overcome. Lack of knowledge at small distances is a 
barrier to understanding the response of small, relatively insensitive detectors, while at large distances this lack is a barrier to the accurate evaluation of cross sections in the thin-down region.

More recent work has been done by Metting ${ }^{(32-34)}$ and Toburen ${ }^{(35,36)}$ with 13.0 , 13.8 and 17.2 MeV/amu Ge as projectiles, with $\mathrm{U}$ at $5.9 \mathrm{MeV} / \mathrm{amu}$, and with $\mathrm{Fe}$ at $600 \mathrm{MeV} / \mathrm{amu}$. Kanai and Kawachi ${ }^{(37)}$ used $18.3 \mathrm{MeV} / \mathrm{amu} \boldsymbol{\alpha}$ particles.

The work of Kanai and Kawachi is not different in principle from the earlier work of the Baum group, although it differed in detail, for it measured the energy deposited within a cylinder coaxial with the ion's path. The radius was scaled by varying gas pressure. The average radial dose as a function of distance from the ion's path was found by successive differences in the energy deposited in the cylinder at incremental pressures. This procedure, like that of the Brookhaven group, yielded the average radial dose.

Metting's work was conceptually different. She sought to measure the fluctuations in energy deposition in small proportional counters at different radial distances from the ion's path using equivalent micrometer-sized volumes. When these measurements were processed so as to yield the average radial dose distribution, they were found to be in excellent agreement with earlier measurements and with our analytical model.

There had been questions as to the validity of the concept of effective charge as applied to this work. As seen in the reference frame in which the projectile is at rest, electrons in the target that pass close to the projectile nucleus are partly shielded from the outermost electrons carried by the projectile, and hence should experience a different "effective charge" than that experienced by electrons passing far from the nucleus. ${ }^{(38)}$ This anticipated problem did not arise in Metting's measurements. The measurements were consistent with the use of standard effective charge formulas.

\section{Other Theory}

The earliest calculations of the radial distribution of dose known to us were published by Hutchinson ${ }^{(39)}$ in connection with his effort to supplement the associated volume model of Lea in relation to the measured cross sections for the inactivation of dry enzymes and viruses. Three different calculations were made, one of which preceded the later calculation made independently by Butts and Katz in connection with their theory of RBE for the inactivation of dry enzymes and viruses. To our knowledge, no quantitative application of these calculations was published by Hutchinson.

In the ensuing years, many calculations of the radial-dose distribution about the path of a heavy ion have been made by different investigators. Two different sorts of data are required. One needs a source function that describes the radial distribution of the primary excitations and ionizations in the medium from the passing ion, and the doubly differential (in both energy and angle) cross section for secondary electron (delta ray) production. Then we must know the manner of energy deposition by the delta rays themselves. The energy deposited by delta rays has been evaluated in the continuous slowing-down approximation and by Monte Carlo methods, using experimental electron collision cross sections for gases, and using mean free paths calculated from optical measurements in liquid water. The results are in reasonable agreement with each other and with such experimental data as exist.

The calculations can be tested against direct measurements of the radial dose distribution in gases. But no direct measurements can be made for the radial dose distribution in condensed matter; yet, it is condensed matter that many of the radiation effects of interest take place. Thus far, the only available tests in condensed matter 
are from the comparison of track theory calculations of the response of detectors to heavy ions, which were made from the calculated radial dose distributions. Even with calculations, as with measurement, there are unresolved problems in calculating the dose very close to the ion's path, (e.g., within a hundred angstroms) and out to the maximum radial penetration of delta rays.

Calculations made of the radial dose distribution in the continuous slowing-down approximation were made by Fain, ${ }^{(40,41)}$ by Chatterjee, ${ }^{(42,43)}$ by Hansen, ${ }^{(44)}$ and by Zhang et al. ${ }^{(45)}$ In some cases, it was assumed that there was a uniform "core" of energy dissipation close to the ion's path whose radius was given by the Bohr adiabatic approximation, ${ }^{(5,46)}$ an inference of questionable validity.

Other calculations have been made by Monte Carlo methods, using extensive compilations of electron interaction cross sections measured in gases, ${ }^{(47,48)}$ or by using optical data to generate electron mean-free paths in water at Oak Ridge. ${ }^{(49,50)}$ More recently, a direct comparison has been provided between radial dose distributions in gas-phase and liquid-phase water using Monte Carlo transport techniques (Varma and Zaider, private communications, 1990).

A point of interest is the possibility of using a description of the track in terms of its radial dose distribution as a substitute for its microdosimetric description. A detailed study of this problem ${ }^{(51)}$ indicated that the critical parameter is the ratio between the diameter of the microdosimetric volume and the maximum lateral extension of the track (accurate results obtained when this ratio is significantly larger than 1). Radiobiological quantities, however, such as RBE appear to be described equally well in terms of either one of these two important field descriptors.

Our present best estimates of the radial dose distribution in water and other media is based on the Oak Ridge model ${ }^{(50)}$ as presented in Fig. 1. The calculation, neglecting primary excitations, is based on the Rutherford formula, the approximation that delta rays are normally ejected, and a power law approximation to the range of electrons in aluminum. This approximation is taken in two segments. One fits the data well below $1 \mathrm{keV}$; the other fits much of the data reasonably well for electrons above $1 \mathrm{keV}$. In the Rutherford formula, an adjusted ionization potential is assumed for the medium ( $10 \mathrm{eV}$ for water) so that the integrated radial dose is finite. A multiplicative correction is then applied to this formula to agree with Hamm's Monte Carlo calculations, using the Oak Ridge formulation, of radial dose in water. This yields a bump in the plotted curve at radial distances below $10 \mathrm{~nm}$, presumably accommodating the primary excitations. The Waligorski formulation is an extension of the formulation of Zhang et al., ${ }^{(45)}$ which ignores the energy deposited by primary excitations and ionizations, to fit Hamm's Monte Carlo calculation and adjusted to agree with tabular values of the stopping power of protons in water at proton energies from 0.1 to $1000 \mathrm{meV}$.

A series of graphs displays the relation between the formulas of Waligorski and the experimental measurements in gases. In Fig. 2 we compare the calculations of Zhang ${ }^{(45)}$ and of Waligorski ${ }^{(50)}$ (labeled "this work") to measurements with protons, deuterons, and alpha particles by Menzel and $\mathrm{Booz}^{(52)}$ and Wingate and Baum. ${ }^{(31)}$ In Fig. 3 we compare these calculations to the work of Varma and co-workers ${ }^{(53)}$ for $\mathrm{O}, \mathrm{Br}$, and $\mathrm{I}$ from the Tandem Van de Graaf accelerator and for $\mathrm{He}$ and $\mathrm{Ne}$ from the Berkeley BEVALAC accelerator. Also shown in Fig. 3 are the results of Monte Carlo calculations by Paretzke for $930 \mathrm{MeV}$ He ions.

In Fig. 4 we compare the Zhang and the Waligorski ${ }^{(45,50)}$ calculations to the calculations by Fain, ${ }^{(40)}$ made in the continuous slowing-down approximation, and to 
Radial dose distribution:

$$
\begin{array}{ll}
D_{1}(t)=\frac{\mathrm{Ne}^{4} Z^{* 2}}{\alpha m c^{2} B^{2} t} \cdot \frac{(1-((t+\theta) /(T+\theta)))^{1 / \alpha}}{t+\theta} \\
\theta=R(I) & I=10 \mathrm{eV} \\
T=R(H) & W=2 m c^{2} B^{2}\left(1-B^{2}\right)^{-1 / 2}
\end{array}
$$

Electron range-energy relation for aluminium:

$$
\begin{aligned}
R & =k \cdot w^{\alpha} \\
k & =6 \times 10^{-6} \mathrm{~g} \cdot \mathrm{cm}^{2} \cdot \mathrm{keV}^{-\alpha} \\
w<1 \mathrm{keV} & \alpha=1.079 \text { for ion } B \leqslant 0.03 \\
w>1 \mathrm{keV} \quad \alpha & =1.667 \text { for ion } B>0.03
\end{aligned}
$$

Delta ray distribution:

$$
d n=\frac{2 \pi N e^{4} Z^{\star 2}}{m c^{2} B^{2}} \cdot \frac{d w}{(w+I)^{2}}
$$

Constant for liquid water:

$$
N=\frac{2 \pi \mathrm{e}^{4}}{\mathrm{mc}^{2} \mathrm{~B}^{2}}=1.369 \times 10^{-14} \mathrm{~J} \cdot \mathrm{cm}^{-1}=8.5 \mathrm{keV} \cdot \mathrm{mm}^{-1}
$$

Effective charge:

$$
Z^{\star}=Z\left(1-\exp \left(-125 \cdot \beta \cdot Z^{-2 / 3}\right)\right)
$$

Corrected radial dose distribution:

$$
\begin{aligned}
& D_{2}(t)=D_{1}(t) \cdot(1+K(t)) \\
& K(t)=A \cdot((t-B) / C) \cdot \exp (-(t-B) / C) \\
& A=8 \cdot B^{1 / 3} \text { for } B \leqslant 0.03 \\
& A=19 \cdot B^{1 / 3} \text { for } B>0.03 \\
& B=0.1 \mathrm{~nm} \\
& C=1.5 \mathrm{~nm}+B .5 \mathrm{~nm} \\
& K(t)=0 \text { for } t<B
\end{aligned}
$$

Figure 1. Equations for the radial dose distribution in liquid water, from Waligorski et al. ${ }^{(50)}$

Zaider's Monte Carlo calculations, ${ }^{(48)}$ which were made using experimental gas-phase cross sections. In Fig. 5 we compare the Waligorski calculation to measurements by Kanai $^{(37)}$ for alpha particles.

In all cases, our calculated radial dose is scaled from the formulas for protons by use of the effective charge according to Eq. 10 in Fig. 1.

Note that in all cases, the data do not reflect the "bump" in the Waligorski curves (this work). Note also that the data are very sparse close to, and far from, the ion's path. Nevertheless, it is quite remarkable that expressions calculated from a wide variety of source data for condensed matter are in such good agreement with measurements and Monte Carlo calculations made for gases. It is also remarkable that the oversimplified calculation, which totally neglects the angular distribution of ejected 

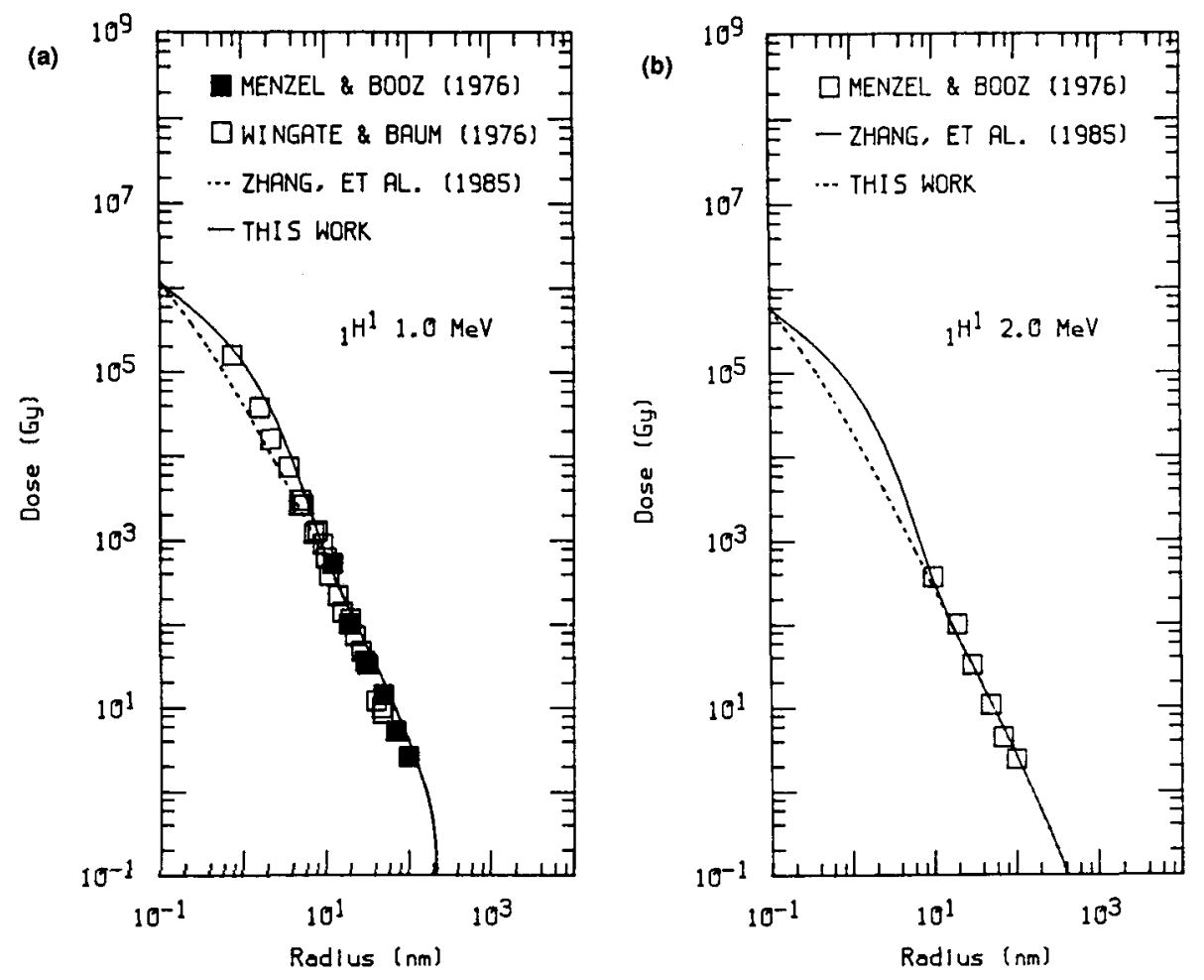

Figure 2. Data of Menzel and $\mathrm{Booz}^{(52)}$ and of Wingate and Baum ${ }^{(31)}$ compared to calculations from Zhang et al. ${ }^{(45)}$ and of Waligorski et al.., ${ }^{(50)}$ here labeled "this work". 


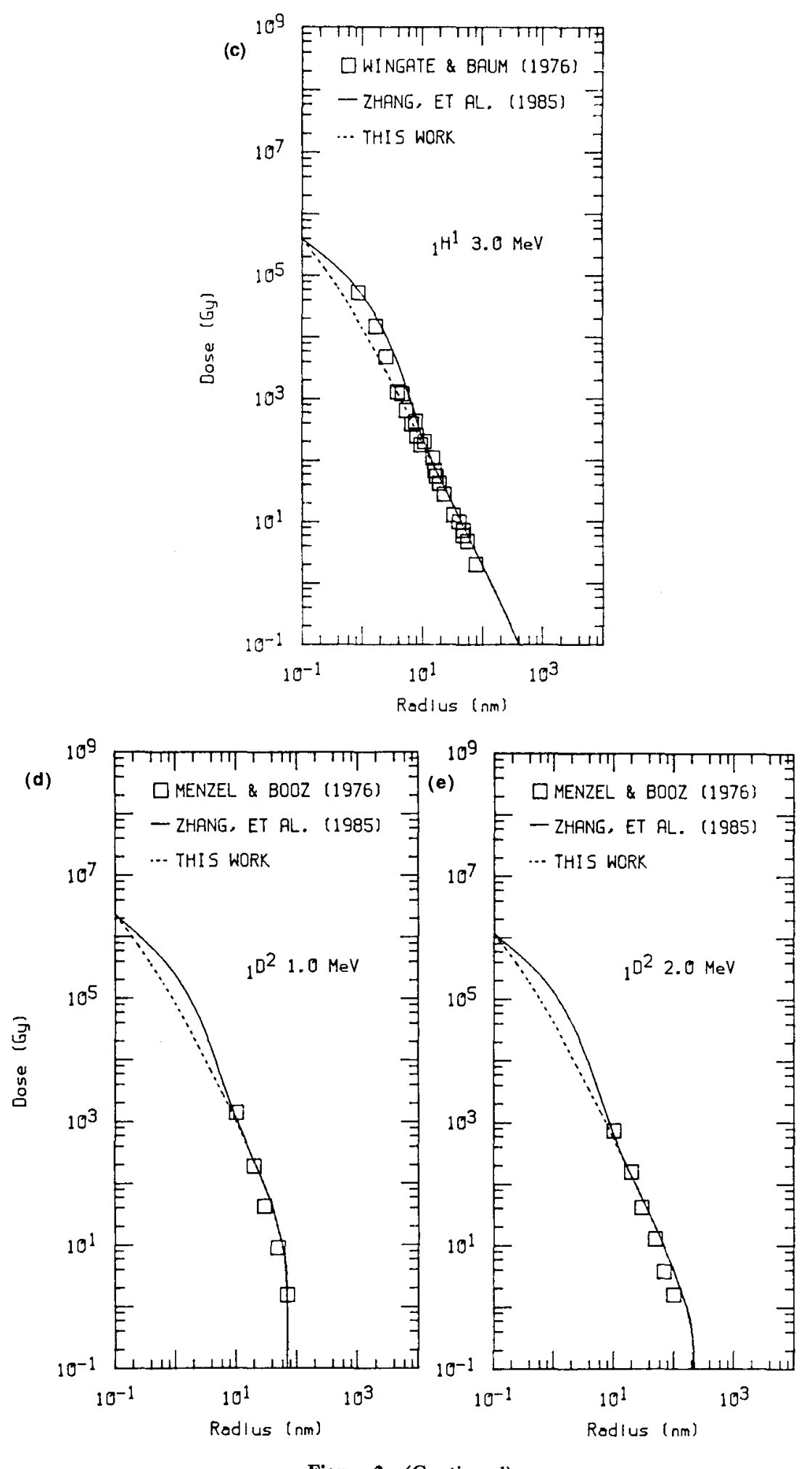

Figure 2. (Continued) 

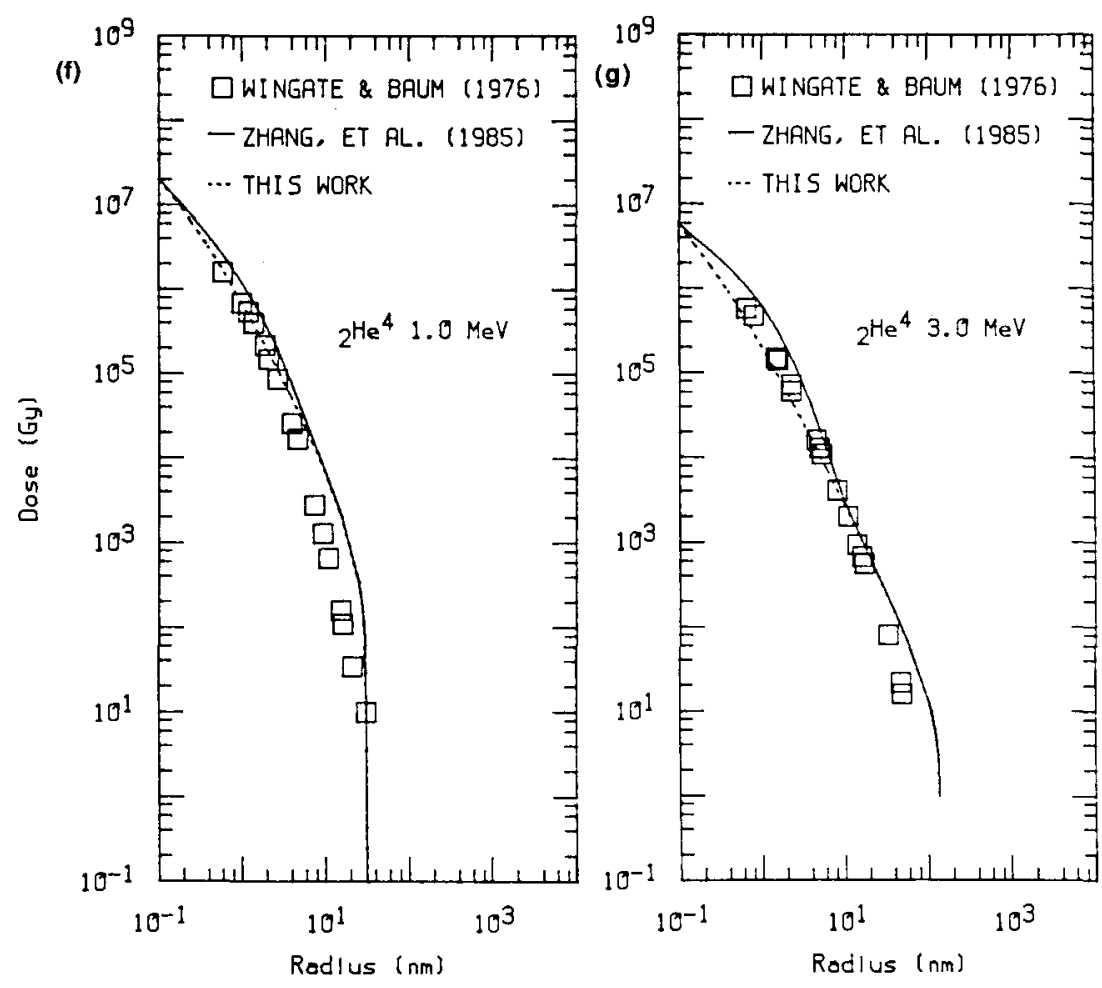

Figure 2. (Continued) 


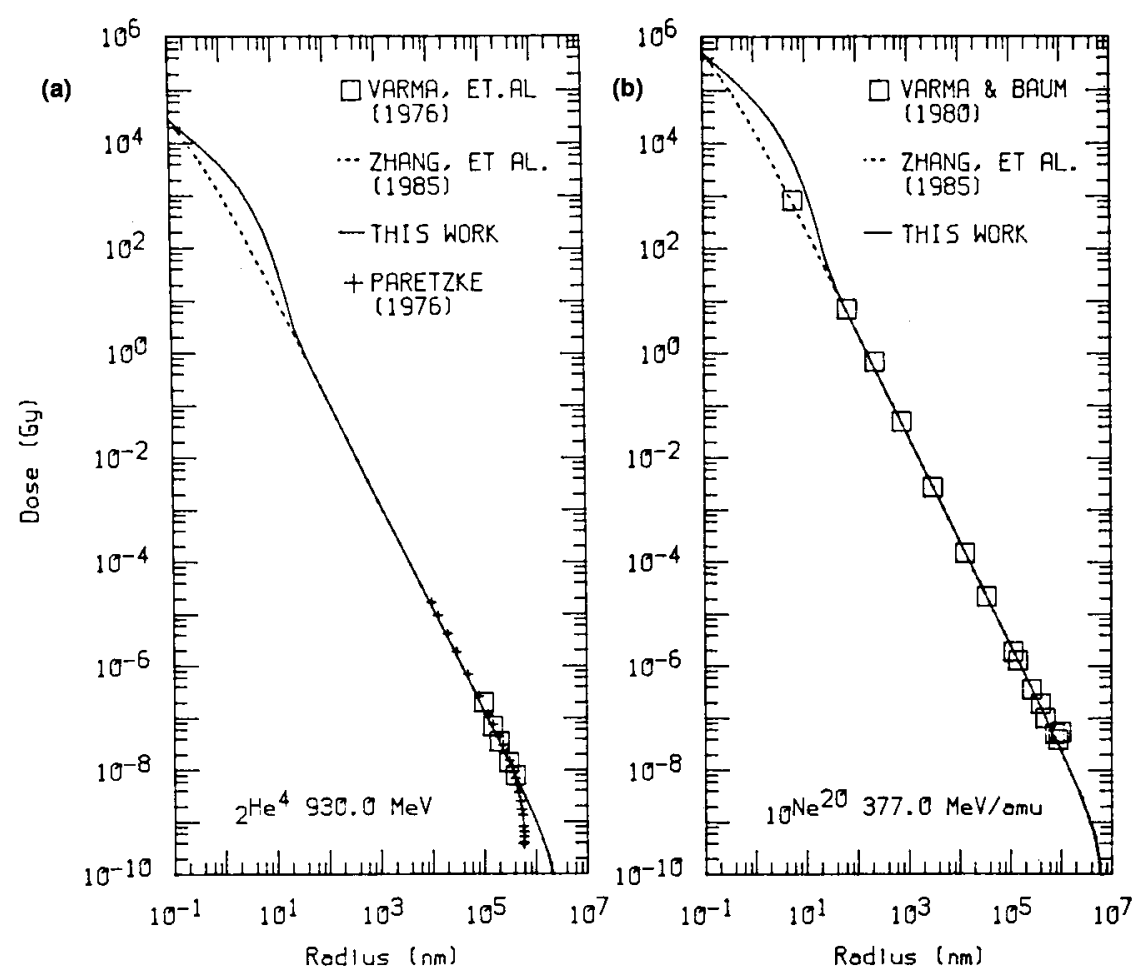

Figure 3. Data of Varma et al. ${ }^{(53)}$ compared to calculations from Zhang ${ }^{(45)}$ and from Waligorski. ${ }^{(50)}$ 

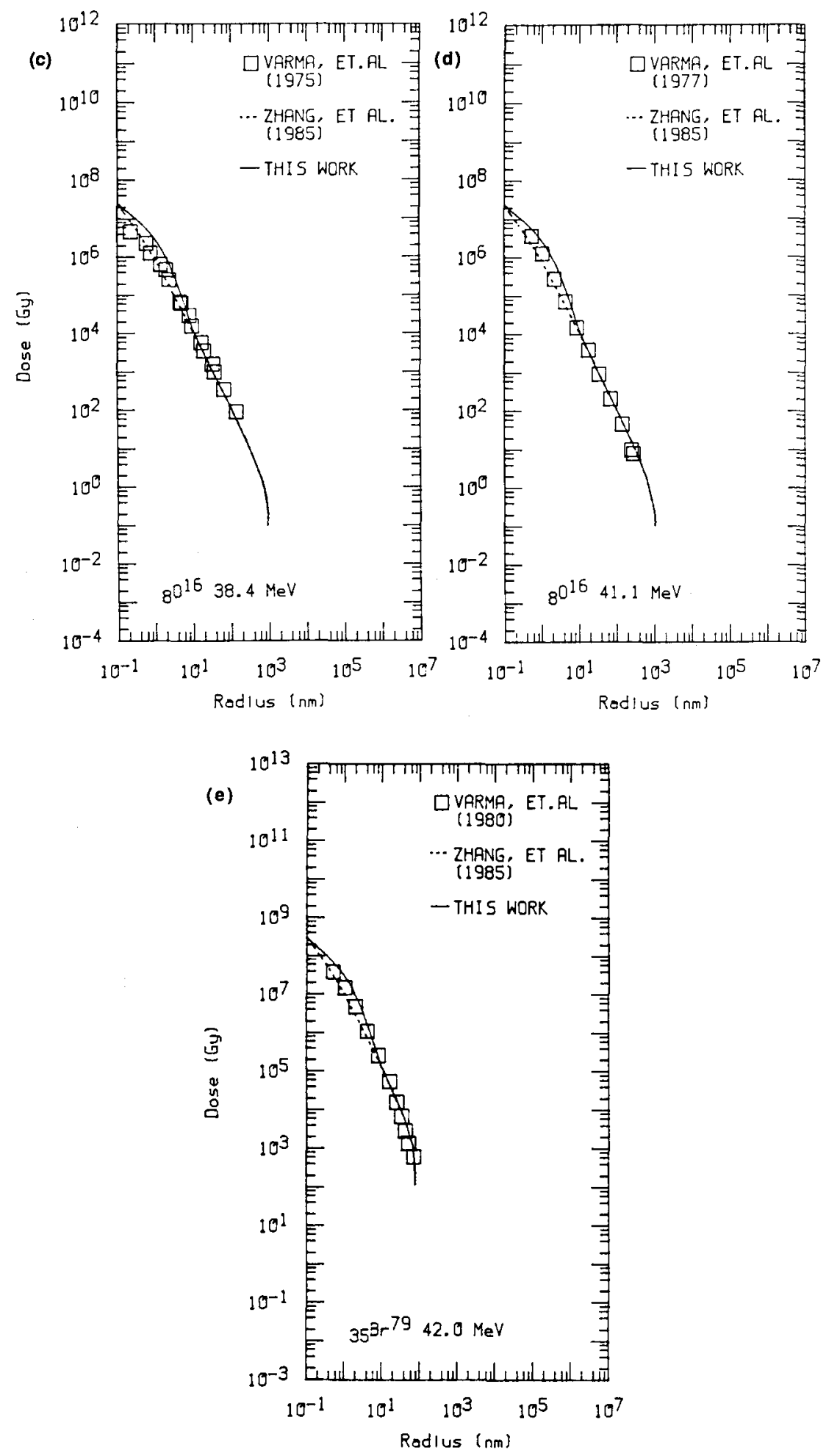

Figure 3. (Continued) 


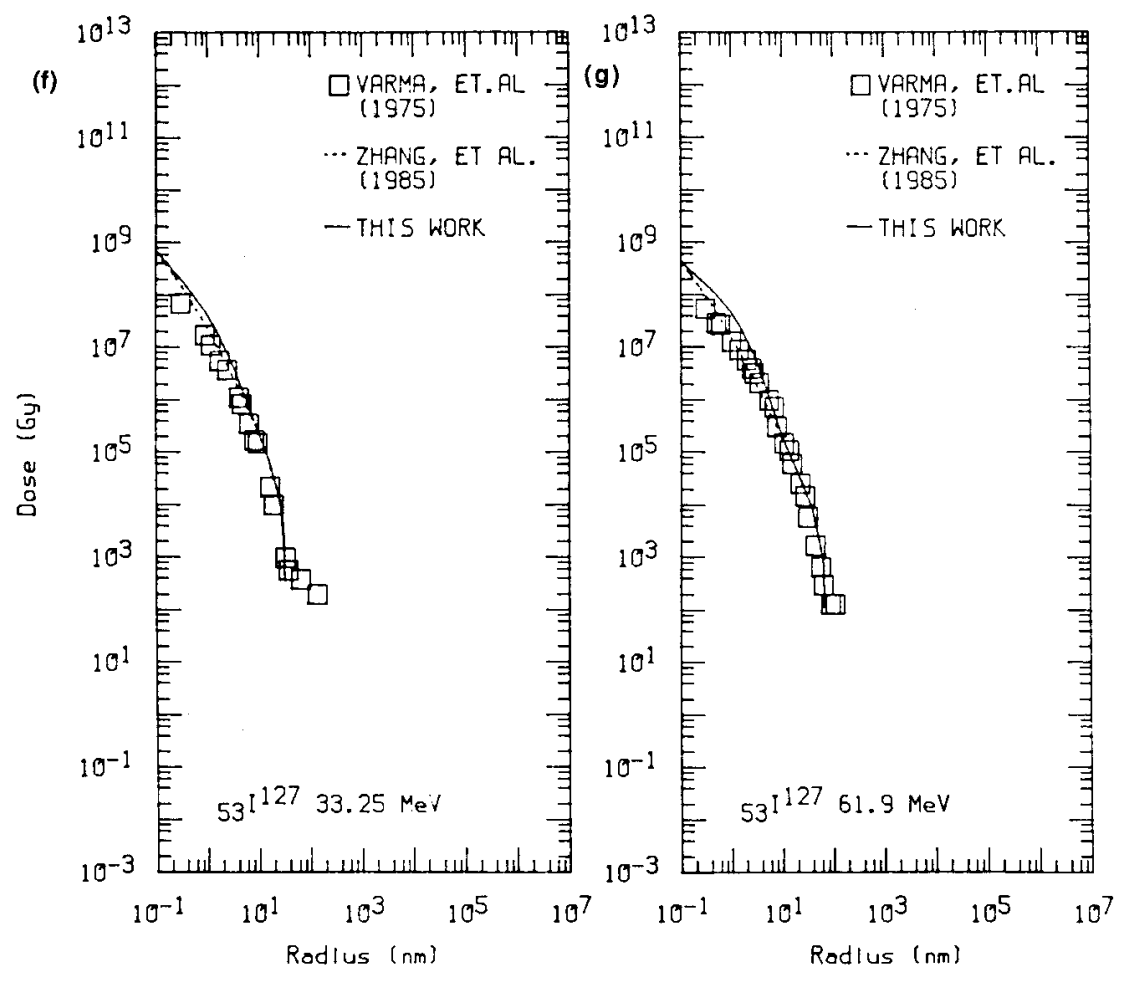

Figure 3. (Continued) 

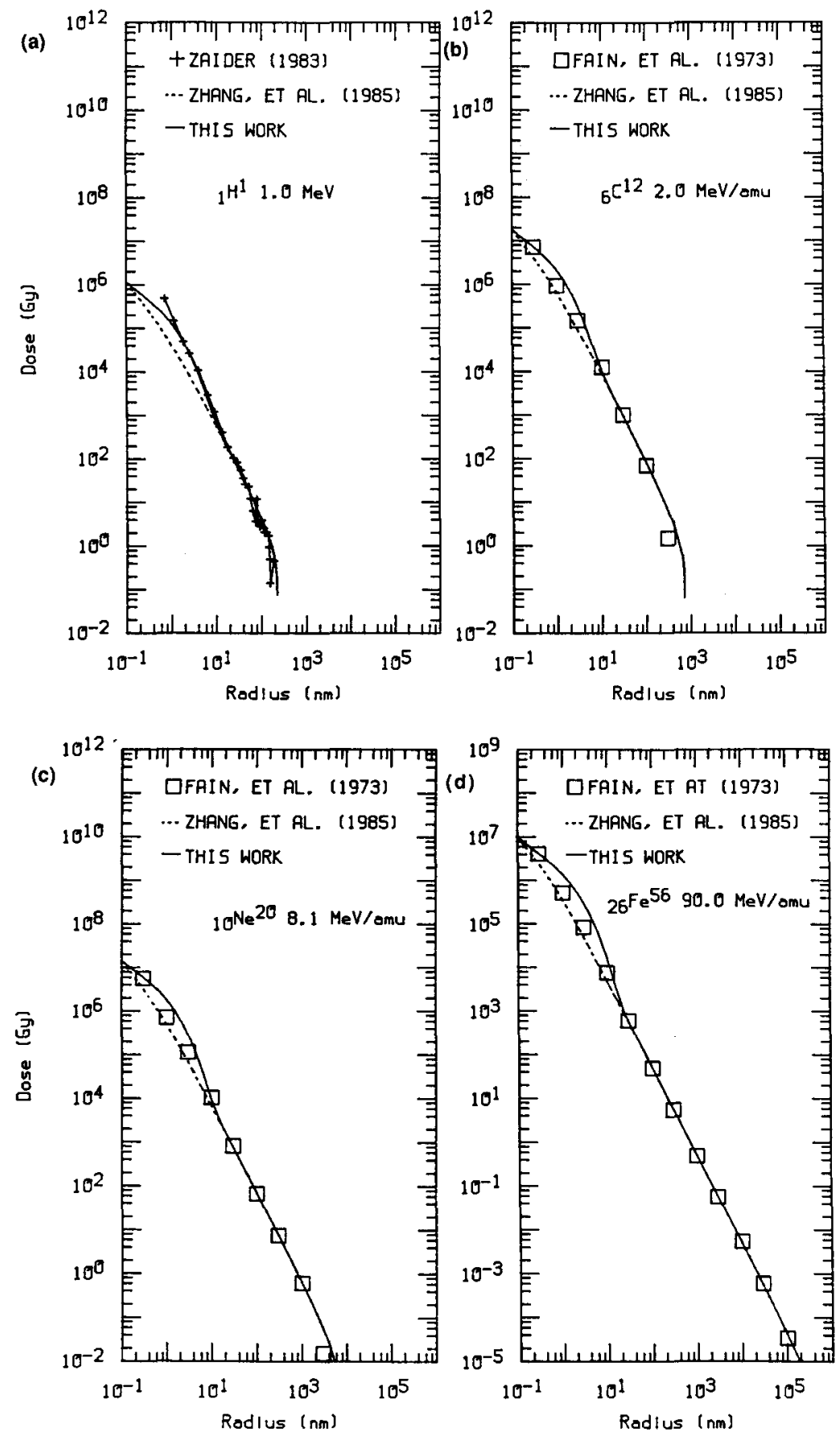

Figure 4. Calculations of Zaider et al. ${ }^{(48)}$ and of Fain et al. ${ }^{(40,41)}$ compared to the calculations of Zhang $^{(45)}$ and of Waligorski. ${ }^{(50)}$ 


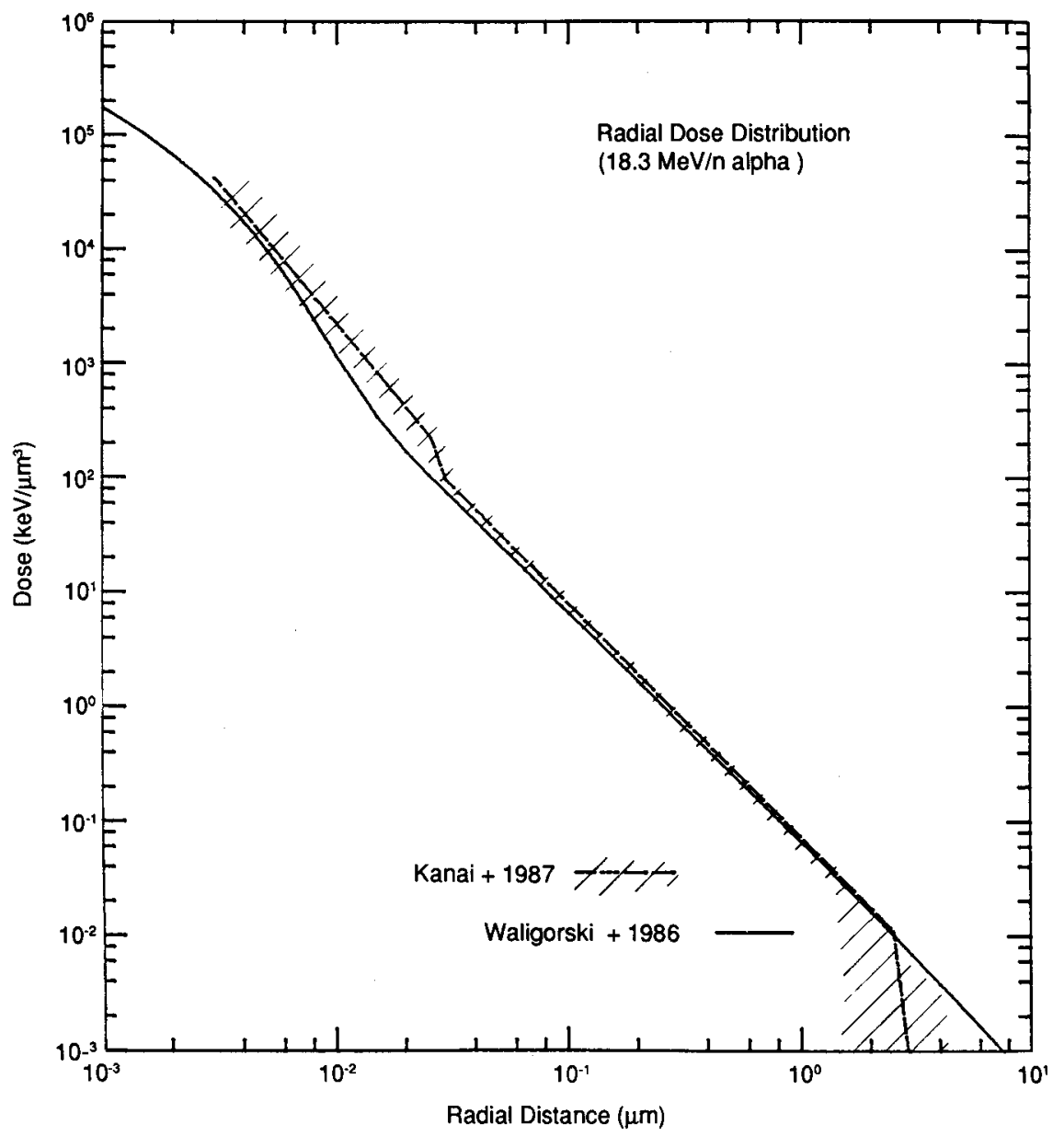

Figure 5. Measurements of Kanai and Kawachi ${ }^{(37)}$ compared to the calculation of Waligorski. ${ }^{(50)}$

electrons and the difference in ionization potentials of the different shells from which the electrons are ejected, can yield such good results, not only for the radial dose distribution, but also for the subsequent calculations of cross sections.

We can anticipate that both calculations and measurements of the radial distribution of dose will continue, and that our knowledge concerning this important component of the study of the effects of high LET radiation will continually improve. To that end, we note that $\operatorname{Rudd}^{(54)}$ has formulated a user-friendly model for the energy distribution of electrons from proton or electron collisions.

Continuing in the mode of our own oversimplifications, we have recently produced an analytic formulation of the radial dose distribution in several solids of interest in radiation measurement, based on an extension of our formulation for liquid water. ${ }^{(18)}$ We have applied this formulation to the calculation of the response of $\mathrm{NaI}(\mathrm{T} 1)$ and $\mathrm{LiF}$ (TLD100) to energetic heavy ions. ${ }^{(5)}$ In the latter case, we have shown that this model accounts for the observation of "hooks" in the response of this thermoluminescent dosimeter to slowing heavy ions, precisely parallel to similar observations in radiobiology. ${ }^{(56)}$ One value of a global parametric model of radiation effects is that parallel analyses of similar events in different detectors enable a correct attribution of 
these hooks to their common origin, the radial dose distribution from delta rays rather than to some imaginative mechanistic models of the behavior of particular detectors, or of biological cells.

\section{Acknowledgment}

This work was supported by the U.S. Department of Energy.

\section{References}

1. R. Katz and J. J. Butts. On the Width of Heavy Ion Tracks in Emulsion. In Proc. of the 5th Int. Conf. on Nuclear Photography. CERN 65-4, Vol. 2, IX-48 (1965).

2. R. Katz and J. J. Butts. Width of Ion and Monopole Tracks in Emulsion. Phys. Rev. 137:B198-B203 (1965).

3. J. P. Lonchamp. Contribution a l'Étude Methodologique des Emulsions Photographiques Utilisées en Physique Nucleaire. Ann. de Phys. 10:201-258 (1955).

4. P. G. Bizetti and M. Della Corte. On the Thinning Down of Tracks of Heavy Nuclei in Nuclear Emulsions. Nuovo Cimento 9:317-333 (1959).

5. J. Orear, A. H. Rosenfeld, and R. A. Schluter. Energy Loss by Charged Particles, Chapter IIA in Nuclear Physics, a Course Given by Enrico Fermi, pp. 27-34. University of Chicago Press, Chicago (1949).

6. W. H. Barkas. Nuclear Research Emulsions 1. Academic Press, New York (1963).

7. J. J. Butts and R. Katz. Theory of RBE for Heavy Ion Bombardment of Dry Enzymes and Viruses. Radiat. Res. 30:855-871 (1967).

8. A. M. Kellerer and H. H. Rossi. The Theory of Dual Radiation Action. Curr. Topics Radiat. Res. Q1. 8:85-158 (1972).

9. A. M. Kellerer and H. H. Rossi. A Generalized Formulation of Dual Radiation Action. Radiat. Res. 75:471-488 (1978).

10. E. J. Kobetich and R. Katz. Energy Deposition by Electron Beams and Delta Rays. Phys. Rev. 170:391-396 (1968).

11. E. J. Kobetich and R. Katz. Electron Energy Dissipation. Nucl. Instr. Meth. 71:226-230 (1968).

12. R. Katz and E. J. Kobetich. Response of Nuclear Emulsion to Electron Beams. Nucl. Instr. Meth. 79:320-324 (1970).

13. R. Katz and E. J. Kobetich. Particle Tracks in Emulsion. Phys. Rev. 186:344-351 (1969).

14. C. F. Powell, P. H. Fowler, and D. H. Perkins. The Study of Elementary Particles by the Photographic Method. Pergamon Press, New York (1959).

15. P. H. Fowler, R. A. Adams, V. G. Cowen, and J. M. Kidd. The Charge Spectrum of Very Heavy Cosmic Ray Nuclei. Proc. Roy. Soc. A. 301:39-45 (1967).

16. R. Katz and F. E. Pinkerton. Response of Nuclear Emulsions to Ionizing Radiations. Nucl. Instr. Meth. 130:105-119 (1975).

17. R. Katz, A. S-F. Li, Y. L. Chang, R. L. Rosman, and E. V. Benton. Tracks of Argon Ions in Ilford K-Series Nuclear Track Detectors. The Nucleus (Pakistan) 20:17-20 (1983).

18. R. Katz, K. S. Loh, Luo Daling, and G. R. Huang. An Analytic Representation of the Radial Distribution of Dose from Energetic Heavy Ions in Water, $\mathrm{Si}, \mathrm{NaI}$, and $\mathrm{SiO}_{2}$. Radiat. Effects and Defects in Soiids 114:15-20 (1990).

19. M. Jensen, L. Larsson, O. Mathiesen, and R. Rosander. Experimental and Theoretical Absorptance Profiles of Tracks of Fast Heavy Ions in Nuclear Emulsion. Physica Scripta 13:65-74 (1976).

20. M. Jensen and O. Mathiesen. Measured and Calculated Absorptance of Ttacks of Fast Heavy Ions in Ilford G.5 Nuclear Emulsion. Physica Scripta 13:75-82 (1976).

21. S. Behrnetz. Application of Track Formation Theory to Calibration of Photometric Measurements on Cosmic Ray Tracks in Nuclear Emulsion. Nucl. Instr. Meth. 133:113-119 (1976).

22. R. Katz. Photometric Measurements of Thin Tracks in Nuclear Emulsion. Nucl. Instr. Meth. 169:257-259 (1979).

23. J. W. Baum. Comparison of Distance and Energy Related Restricted Energy Transfer to Heavy Particles with 0.25 to $1100 \mathrm{MeV} / \mathrm{amu}$. Brookhaven National Laboratory (1967).

24. J. W. Baum, S. L. Stone, and A. V. Kuehner. Radial Distribution of Dose Along Heavy Ion Tracks, LET. In Proc. Symp. Microdosim., H. G. Ebert, ed., pp. 269-281. Ispra, Italy (1968).

25. J. W. Baum, M. N. Varma, C. L. Wingate, H. G. Paretzke, and A. V. Kuehner. Nanometer Dosimetry of Heavy Ion Tracks. In Proc. 4th Symp. Microdosim., J. Booz, H. G. Ebert, R. Eikel, and A. Waker, eds., 1:93-112. EUR 5122 d-e-f, Verbania Pallanza, Italy (1974). 
26. M. N. Varma, J. W. Baum, and A. V. Kuehner. Energy Deposition by Heavy Ions in a "Tissue Equivalent" Gas. Radiat. Res. 62:1-11 (1975).

27. M. N. Varma, H. G. Paretzke, J. W. Baum, J. T. Lyman, and J. Howard. Dose as a Function of Radial Distance from a $930 \mathrm{MeV}^{4} \mathrm{He}$ Ion Beam. In Proc. 5th Symp. Microdosim., J. Booz, H. G. Ebert, and B.G.R. Smith, eds., 1:75-79. EUR 5452 d-c-f, Verbania Pallanza, Italy (1976).

28. M. N. Varma, J. W. Baum, and A. V. Kuehner. Radial Dose, LET and $\mathrm{W}$ for ${ }^{16} \mathrm{O}$ Ions in the $\mathrm{N}_{2}$ and Tissue Equivalent Gases. Radiat. Res. 70:511-518 (1977).

29. M. N. Varma and J. W. Baum. Energy Deposition in Nanometer Regions by $377 \mathrm{MeV} /$ nucleon ${ }^{20}$ Ne Ions. Radiat. Res. 81:355-363 (1980).

30. M. N. Varma. Review of Radial Dose Measurement Technique and Data. Nucl. Tracks. Radiat. Meas. 16:135-139 (1989).

31. C. L. Wingate and J. W. Baum. Measured Radial Distribution of Dose and LET for Alpha and Proton Beams in Hydrogen and Tissue-Equivalent Gas. Radiat. Res. 65:1-17 (1976).

32. N. F. Metting. Measurement of Energy Deposition Near High Energy Heavy Ion Tracks. Master's Thesis, University of Washington. Pacific Northwest Laboratory, Richland, Washington (1986).

33. N. F. Metting. A Comparison of Microscopic Dose with Average Dose Near High Energy Ions. Nucl. Instr. Meth. in Phys. Res. B24/25:1050-1053 (1987).

34. N. F. Metting, H. H. Rossi, L. A. Braby, P. J. Kliauga, J. Howard, M. Zaider, W. Schimmerling, M. Wong, and M. Rapkin. Microdosimetry Near the Trajectory of High Energy Ions. Radiat. Res. 116:183-195 (1988).

35. L. H. Toburen, N. F. Metting, and L. A. Braby. Spatial Patterns of Ionization in Charged Particle Tracks. Nucl. Instr. Meth. in Phys. Res. B40/41:1275-1278 (1989).

36. L. H. Toburen, L. A. Braby, N. F. Metting, G. Kraft, H. Schmidt-Bocking, R. Dorner, and R. Seip. Radial Distributions of Energy Deposited Along Charged Particle Tracks. In 10th Symposium on Microdosimetry, Rome, May 1989 (1990).

37. T. Kanai and K. Kawachi. Radial Dose Distribution for $18.3 \mathrm{MeV} / \mathrm{n}$ Alpha Beams in Tissue Equivalent Gas. Radiat. Res. 112:426-435 (1987).

38. L. H. Toburen, W. E. Wilson, and R. J. Popowich. Secondary Electron Emission from Ionization of Water Vapor by 0.3 to $2.0 \mathrm{MeV} \mathrm{He}+$ and He2+ Ions. Radiat. Res. 82:27-44 (1980).

39. F. Hutchinson. The Interaction of Primary Cosmic Rays with Matter and Tissue. In Symposium on Medical and Biological Aspects of the Energies of Space, P. A. Campbell, ed., Columbia University Press, New York (1961).

40. J. Fain, M. Monnin, and M. Montret. Energy Density Deposited by a Heavy Ion Around Its Path. In Proc. 4th Symp. Microdosim. J. Booz, H. G. Ebert, R. Eikel, and A. Waker, eds., pp. 169-188. Verbania-Pallanza, Italy (1974).

41. J. Fain, M. Monnin, and M. Montret. Spatial Energy Distribution Around Heavy Ion Paths. Radiat. Res. 57:379-389 (1974).

42. A. Chatterjee, H. D. Maccabee, and C. A. Tobias. Radial Cut-Off LET and Radial Cut-Off Dose Calculations for Heavy Charged Particles in Water. Radiat. Res. 54:479-494 (1973).

43. A. Chatterjee and H. J. Schaefer. Microdosimetric Structure of Heavy Ion Tracks in Tissue. Radiat. Environ. Biophys. 13:215-227 (1976).

44. J. W. Hansen and K. J. Olsen. Experimental and Calculated Response of a Radiochromic Dye Film Dosimeter to High-LET Radiations. Radiat. Res. 97:1-15 (1984).

45. C. Zhang, D. E. Dunn, and R. Katz. Radial Distribution of Dose and Cross Section for the Inactivation of Dry Enzymes and Viruses. Radiat. Prot. Dosim. 13:215-218 (1985).

46. W. Brandt and R. H. Ritchie. Primary Processes in the Physical Stage. In Physical Mechanisms in Radiation Biology, R. D. Cooper and R. W. Wood, eds., pp. 20-46. U.S. Atomic Energy Commission CONF-721001 (1974).

47. H. G. Paretzke, G. Leuthold, G. Burger, and W. Jacobi. Approaches to Physical Track Structure Calculations. In Proc. 4th Symp. Microdosim., J. Booz, H. Ebert, R. Eickel, and A. Waker, eds., 1:75-79. EUR 5122 d-e-f, Verbania Pallanza, Italy (1976).

48. M. Zaider, D. J. Brenner, and W. E. Wilson. The Applications of Track Calculations to Radiobiology 1. Monte Carlo Simulation of Proton Tracks. Radiat. Res. 95:231-242 (1983).

49. R. H. Ritchie, R. N. Hamm, J. E. Turner, H. A. Wright, J. C. Ashley, and G. J. Basbas. Physical Aspects of Charged Particle Track Structure. Nucl. Tracks Radiat. Meas. 16:141-155 (1989).

50. M.P.R. Waligorzki, R. N. Hamm, and R. Katz. Radial Distribution of Dose Around the Path of a Heavy Ion in Liquid Water. Nucl. Tracks Radiat. Meas. 11:309-319 (1986).

51. N. M. Varma and M. Zaider. The Radial Dose Distribution As a Microdosimetric Tool. Radiat. Prot. Dosim. 31:155-160 (1990). 
52. H. G. Menzel and J. Booz. Measurement of Radial Energy Deposition Spectra for Protons and Deuterons in Tissue Equivalent Gas. In Sth Symposium on Microdosimetry, J. Booz, H. G. Ebert, B.G.R. Smith, eds. Commission of the European Communities, Luxembourg (1976).

53. M. N. Varma, J. W. Baum, and A. V. Kuehner. Stopping Power and Radial Dose Distribution for $42 \mathrm{MeV}$ Bromine Ions. Phys. Med. Biol. 25:651-656 (1980).

54. M. E. Rudd. User Friendly Model for the Energy Distribution of Electrons from Proton or Electron Collisions. Nucl. Tracks Radiat. Meas. 16:213-218 (1989).

55. R. Katz and Luo Daling. Response of $\mathrm{NaI}(\mathrm{T} 1)$ and $\mathrm{TLD}(100)$ to Energetic Heavy Ions. 9th Int. Conf. on Solid State Dosimetry, Vienna 1989 (in press).

56. R. Katz, E. D. Dunn, and G. L. Sinclair. Thindown in Radiobiology. Radiat. Prot. Dosimetry 13:281-284 (1985).

57. R. Katz. Cross Section. Appl. Radiat. Isot. 41:563-567 (1990).

\section{Discussion}

Ward: What is the range of energies per grain that we were looking at in these discussions?

Katz: It could be something like $100 \mathrm{eV}$. It might be much more than that. Insensitive materials might take much more than that. The most sensitive, I think, would be something between $100 \mathrm{eV}$ and a keV. The size is gram comparable to the radius of a cell when you take the density difference into account.

Ward: How do you account for this? If it takes $100 \mathrm{eV}$ to a $\mathrm{keV}$ to produce a grain, how does this relate to the molecules and the radicals in water or biological media?

Katz: I don't think you make that kind of comparison. What you can see from this is how large the brush of delta rays is. You have an illustration here of what a typical track looks like and how the character of the track changes with the sensitivity of the material. When it was suggested that we might be able to actually see a track in cells, I thought that would be wonderful, because then we wouldn't make all these foolish statements about the importance of LET, and we wouldn't talk quality factor nonsense. We would see what was going on instead of inferring from the prejudices of our past.

Zaider: The associated volume concept contains the radial distribution as a particular case, and the track contains all the cases that are associated with the track, e.g. larger than the target, the same size as the target, or smaller than the target. So I don't think that you should call this a false concept; it is not a mistaken concept. It is more powerful concept than is radial distribution, and the reason for that (correct me if you think I am wrong) is that from the associated volume concept, you can produce radial distributions. You cannot go back.

Katz: The associated volume concept was the original motivation for experiments on dry enzymes and viruses. That concept produced data in the form of cross sections attributed to the sizes of the enzymes and viruses as corrected by Lea's correction, which turned out to be wrong by orders of magnitude. The reason that they were wrong by orders of magnitude is what you see in these tracks.

Zaider: Because it involved radial distribution. But not because the concept is wrong.

Katz: My view is that the concept is wrong if it gives you wrong measurements or wrong calculations.

Moolgavkar: What do you mean by the cross section for enzyme inactivation?

Katz: This is the way that it is measured. You have a beam of particle striking a layer of enzymes or viruses, and you measure the surviving fraction, which declines exponentially and is represented as $\mathrm{e}^{-\sigma \mathrm{F}}$, where $\mathrm{F}$ is the fluence and $\sigma$ is a probability. A cross section is always a probability.

Moolgavkar: Okay. It is a matter of semantics here. 
Katz: No, no. Physical cross sections are probabilities, nothing else.

Moolgavkar: All this exponential behavior, to what does it apply?

Katz: Oh, when the process is a one-hit process. Well, a one-hit process is one where either a simple event in the target or a single incident particle produces the effect so that you can get this exponential response with heavy particles; even when the response to gamma rays has a great big shoulder, as for mammalian cells.

Moolgavkar: Excuse me, one more question. You are making the assumption here that you already have a large number of particles or whatever so that you have a Poisson approximation for something like a binomial distribution. Do you understand the problem?

Katz: The character of the response is due to both fluctuations in energy deposition to the character of the target. Cross section is the measure of the probability of an effect being observed after an infinite number of identical repeated trials (57).

Curtis: It is the probability per unit of fluence?

Katz: Well, that is what probability means. The probability for a single particle....

Curtis: The reason that this quantity has the units of area is because it is the probability per unit fluence.

Katz: Well, that is the same as saying "per incident particle."

Varma: I want to bring the discussion back to what you talked about with radial dose distribution. What you didn't mention (and it is very important) is that what we should have in the record is the fact that all the data that you showed really are from, say, 10 to 20 angstroms away from the track and to a large extent where you have 90 to 95 percent of the energy deposited. The interesting part might be if you looked at 10 angstroms and above, where about 95 percent of the energy is deposition. Experimentally that is very hard to measure. Theoretically, when Herwig calculates or when PNL people calculate, it takes a tremendous amount of time on computers. The point that we need to make (and I don't know the answer to this) is that dose deposition at a very short range, say at 10 angstroms, is probably very important because the secondary electrons are very high in energy. As Harel pointed out, once you go outside the track, you might have an energy deposition that is very large, which might be doing the damage.

Katz: But they are from single electrons, a single electron passing through an emulsion grain or a microdosimeter.

Varma: But sometimes a single electron produces a large amount of energy deposition and sometimes a small energy deposition.

Katz: I think they produce what single electrons produce. What we are doing is normalizing these responses to single electrons at low doses of gamma rays. Otherwise, this wouldn't work at all. But the whole basis of this is that we have a means of normalization. We have a means of calibrating the effects of small dose. That is why we are not doing anything $a b$ initio. It is all phenomenological. Lest you turn your nose up at phenomenology; keep in mind that if there hadn't been Kepler's phenomenology, there wouldn't be Newton's gravitation, and if there hadn't been Balmer's phenomenology, there wouldn't have been Schröedinger. Phenomenology precedes mechanism very often in physics, so don't turn your nose up at it. It will lead you to mechanistic conceptions more frequently than the other way around. 